\title{
On the Analysis of Data Augmentation Methods for Spectral Imaged Based Heart Sound Classification Using Convolutional Neural Networks
}

George Zhou ( $\sim$ gez4001@med.cornell.edu )

Weill Cornell Medicine

Yunchan Chen

Weill Cornell Medicine

Candace Chien

Weill Cornell Medicine

\section{Research Article}

Keywords: Machine learning, data augmentation, cardiac sound analysis, spectrograms, convolutional neural network, cardiology, healthcare automation

Posted Date: September 20th, 2021

DOl: https://doi.org/10.21203/rs.3.rs-888104/v1

License: (c) (1) This work is licensed under a Creative Commons Attribution 4.0 International License. Read Full License 
1 Title: On the Analysis of Data Augmentation Methods for Spectral Imaged Based Heart

2 Sound Classification using Convolutional Neural Networks

3 Authors: George Zhou ${ }^{1 *}$, Yunchan Chen ${ }^{1}$, Candace Chien ${ }^{1}$

4 Affiliations

51 Weill Cornell Medicine, New York, NY, 10021, USA

6 Corresponding Author: George Zhou, gez4001@med.cornell.edu

7

8

9

10

11

12

13

14

15

16

17

18

19

20

21

22

23

24

25 
27 Background: The application of machine learning to cardiac auscultation has the potential to 28 improve the accuracy and efficiency of both routine and point-of-care screenings. The use of

29 Convolutional Neural Networks (CNN) on heart sound spectrograms in particular has defined

30 state-of-the-art performance. However, the relative paucity of patient data remains a significant

31 barrier to creating models that can adapt to the wide range of between-subject variability. To

32 that end, we examined a CNN model's performance on automated heart sound classification,

33 before and after various forms of data augmentation, and aimed to identify the most optimal

34 augmentation methods for cardiac spectrogram analysis.

35 Results: We built a standard CNN model to classify cardiac sound recordings as either normal

36 or abnormal. The baseline control model achieved an ROC AUC of $0.945 \pm 0.016$. Among the

37 data augmentation techniques explored, horizontal flipping of the spectrogram image improved

38 the model performance the most, with an ROC AUC of $0.957 \pm 0.009$. Principal component

39 analysis color augmentation (PCA) and perturbations of saturation-value (SV) of the hue-

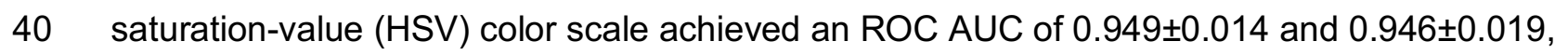

41 respectively. Time and frequency masking resulted in an ROC AUC of $0.948 \pm 0.012$. Pitch

42 shifting, time stretching and compressing, noise injection, vertical flipping, and applying random

43 color filters all negatively impacted model performance.

44 Conclusion: Data augmentation can improve classification accuracy by expanding and

45 diversifying the dataset, which protects against overfitting to random variance. However, data

46 augmentation is necessarily domain specific. For example, methods like noise injection have

47 found success in other areas of automated sound classification, but in the context of cardiac

48 sound analysis, noise injection can mimic the presence of murmurs and worsen model

49 performance. Thus, care should be taken to ensure clinically appropriate forms of data

50 augmentation to avoid negatively impacting model performance. 
51 Key Words: Machine learning, data augmentation, cardiac sound analysis, spectrograms,

52 convolutional neural network, cardiology, healthcare automation

\section{I. Background}

56 Cardiac auscultation has been a core element of the cardiovascular physical exam since the

57 1800s. Sounds produced by the heart reflect its underlying biology and can cue a trained

58 physician to different heart pathologies such as valvular defects or congenital diseases.

59 However, in recent years, cardiac auscultation has been challenged for its diagnostic utility. The

60 decline in accurate cardiac auscultation is a well-documented phenomenon ${ }^{1,2,3}$. For example,

61 internal medicine residents in the US made a correct assessment of auscultation findings only

$6222 \%$ of the time ${ }^{2}$.

64 This has spurred an active area of research in developing suitable machine learning models to

65 classify heart sounds based on recorded phonocardiogram (PCG) signals. Many research

66 groups have published a wide variety of machine learning models to this end. Survey of the

67 existing literature reveals that many different feature extraction methods (Mel-frequency cepstral

68 coefficients ${ }^{4,5,6}$, discrete wavelet transform ${ }^{7,8,9}$, tensor decomposition ${ }^{10}$, sparse coding ${ }^{11}$ ) and

69 classification methods (k-nearest neighbors ${ }^{7}$, support vector machines ${ }^{4,10,11,12}$, hidden Markov

70 models $^{13,14}$, recurrent neural networks ${ }^{15,16}$, convolution neural networks ${ }^{6,17,18}$ ), and their different

71 permutations together have been extensively explored.

72

73 It is generally accepted that bigger datasets result in better machine learning models ${ }^{19,20}$.

74 However, real-world clinical applications is limited by the scarcity of labeled clinical data. This

75 scarcity issue can be attributed to several challenges unique to the medical domain, including:

76 the relative paucity of available clinical databases structured for machine learning research, the 
77 administrative and logistical hurdles associated with collecting and working with patient data and

78 protected health information due to Health Insurance Portability and Accountability Act (HIPAA)

79 laws and Institutional Review Board (IRB) regulations, and finally the time-consuming and

80 expensive nature of properly annotating health data. The gold standard for validating heart

81 sounds is echocardiogram imaging plus the diagnosis from a cardiologist, both of which are

82 costly to obtain. An additional challenge in creating a machine learning model to classify heart

83 sounds is that heart sounds are not actually recorded and stored anywhere in electronic health

84 records (EHR). Mining EHR databases is not an option, meaning heart sounds must be

85 collected and labeled from scratch, one-by-one. Data acquisition is made even harder in times

86 of public health crises, as we have observed with the COVID-19 pandemic, which resulted in

87 drastic reductions in non-emergency patient volumes in clinics across the world.

89 Data augmentation is one solution to the legal limitations and constraints around clinical data.

90 Data augmentation is the process of generating synthetic data from real data, while preserving

91 the class label. In the context of developing machine learning models for heart sound

92 classification, real data means heart sounds collected directly from a patient, whereas synthetic

93 data means artificial heart sounds generated from real heart sounds via various computer-

94 implemented methods.

95

96 The major value add of data augmentation for heart sound classification resides in its ability to

97 significantly expand the size of available training data without the onerous task of having to

98 actually obtain and label a large enough volume of heart sounds. An expanded dataset can

99 improve model performance because the new data created from class-preserving

100 transformations can help the model better learn the unique features that constitute the essence

101 of a class, instead of the random variance that is present within each class. Data augmentation

102 combats overfitting and can help the model make better predictions on unseen data. 
104 Data augmentation is necessarily domain specific, as the applied transformations should reflect 105 realistic variations and preserve the underlying features that distinguish different classes from 106 each other. In other words, the data augmentation should 'make sense' for the task at hand.

107 Two important constraints unique to heart sound spectrograms must be considered in designing 108 effective data augmentation strategies.

110 The first constraint, which we will call the "physiological constraint", is related directly to the 111 phenomenon under study, the heart sound itself. Heart sounds naturally fall within a narrow 112 physiological scope: heart rates are 60-100 beats per minute and the principal frequencies of 113 heart sounds are 20 to $500 \mathrm{~Hz}$. A healthy heart sound can be deconstructed into four main 114 frequency components: S1 (mitral and tricuspid valve closing), systole (ventricles contracting), 115 S2 (aortic and pulmonic valve closing), and diastole (ventricles relaxing). A pathological heart 116 sound has all the same frequency components. The difference between a healthy heart sound 117 and pathological heart sound is that a pathological heart sound will have additional frequency 118 components such as murmurs from valve stenosis or regurgitation, rubs from pericarditis, S3 119 gallops(from increased atrial pressure, as seen in congestive heart failure or dilated 120 cardiomyopathy), or S4 gallops(atrium contracting against stiff ventricle caused by hypertension, 121 pulmonary hypertension, ventricular outflow obstruction, or ischemic heart disease). Of note, an 122 additional sound that can be produced by a healthy heart is the physiological splitting of S2 due 123 to delayed pulmonic valve closing. Thus, the "physiologic constraint" is that any data 124 augmentation method must reflect realistic variations of possible heart sounds and also ensure 125 the presence or absence of additional frequency components is preserved for each individual 126 heart sound or else the distinguishing factor between a normal and abnormal heart sound is lost 127 and the class labels lose their meaning. 
129 The second constraint, which we will call the "spectrogram constraint", is related to the 130 spectrogram image and what it represents. One advantage for using CNN to classify heart 131 sounds is that this converts an audio classification problem into a computer vision problem, 132 which opens the door to the extensive library of data augmentation techniques developed for 133 images. Shorten et al. ${ }^{21}$ published a review article surveying the gamut of image data

134 augmentation techniques that have been researched including flipping, cropping, rotation,

135 translations, color space transformations, kernel filters to sharpen or blur images, mixing 136 images, and random erasing. However, not all image data augmentation techniques will 137 translate appropriately. Although spectrograms are images from a data structure point of view, 138 spectrograms and traditional images have a fundamental difference in terms of what information 139 is conveyed along the $\mathrm{x}$ - and $\mathrm{y}$ - axis. For a traditional image, the axes represent physical 140 distances, while for spectrograms the $\mathrm{x}$-axis represents time and the $\mathrm{y}$-axis represents 141 frequency. Moreover, color also carries a different meaning for traditional images vs 142 spectrogram images. The meaning of color is self-evident for traditional images. For 143 spectrograms, color is an additional dimension that represents decibels, or the loudness and 144 intensity of the heart sound. Thus, the "spectrogram constraint" is that any data augmentation 145 method that operates on the spectrogram as a simple image should correlate with a real-world, 146 physical transformation of the sound.

148 With these constraints in mind, we evaluate common data augmentation techniques at the audio 149 level, including pitch shifting and time stretching/compressing and noise injection, and at the 150 image level, including horizontal flips, vertical flips, hue/brightness transformations, principal 151 component analysis (PCA) color augmentation, random color filters, and time/frequency 152 masking, for classification of heart sounds based on their spectral image. We include 153 augmentation methods that are consistent with and contradict what would be an effective data 154 augmentation method as predicted by our theoretical considerations discussed above to 1) 
examine the individual effectiveness of each augmentation technique on heart sound

156 classification and 2) assess the validity of our theoretical framework.

158 To study the effects of these data augmentation methods on heart sound classification, we

159 separate our experiments into two phases. The first phase is to establish the baseline

160 performance of our $\mathrm{CNN}$ on spectral images of heart sounds. In the second phase, the same

$161 \mathrm{CNN}$ is trained on both real and synthetically generated heart sounds. Model performance with

162 and without data augmentation on the same binary classification task is compared. Each

163 individual data augmentation scheme is carried out in a one-to-one correspondence, meaning

164 for every real heart sound, one synthetic heart sound is generated from it. This doubles the size

165 of the dataset available for training, from $\mathrm{N}$ to $2 \mathrm{~N}$. Figure 1 below shows our study design.
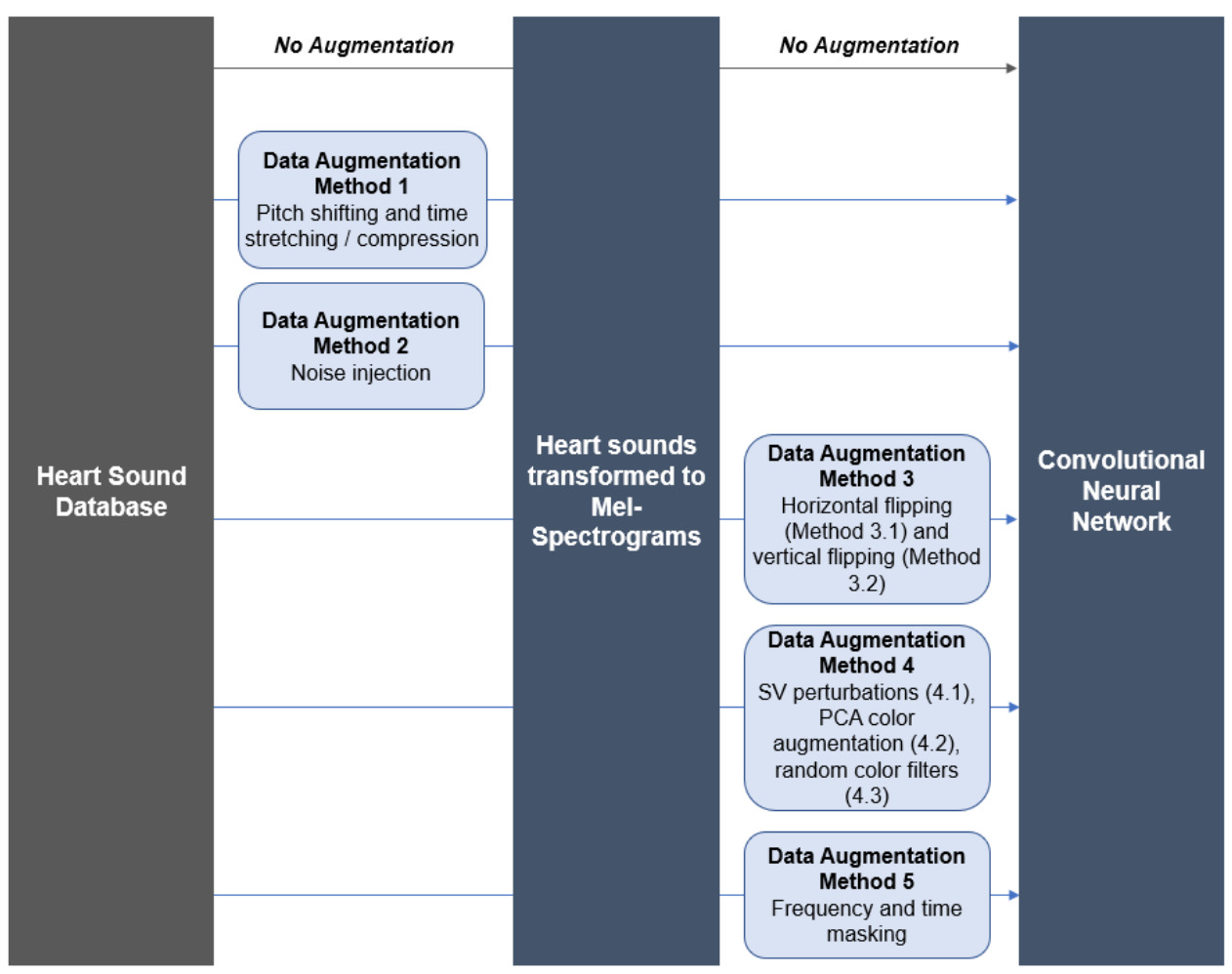

Model 0

Trained on real data

Model 1

Trained on real and

synthetic data from

Method 1

Model 2

Trained on real and

synthetic data from Method 2

\section{Model 3.1 and}

Model 3.2

Trained on real and

synthetic data from

Methods 3.1 and 3.2 respectively

\section{Model 4.1 and} Model 4.2

Trained on real and

synthetic data from

Methods 4.1, 4.2, 4.3 respectively

\section{Model 5}

Trained on real and

synthetic data from Method 4

169 Figure 1: Overview of Study Design 
170 To study the effects of data augmentation on heart sound classification, we established the 171 baseline performance of a machine learning algorithm trained on real heart sound data only 172 (Model 0). We then compared this baseline performance to various models as delineated in the 173 above diagram.

\section{II. Results}

176 Reported metrics are based on a stratified 10-fold cross validation. The folds are created in a

177 consistent way across the different models. This serves to limit any potential variability in model

178 performance that would be due to the underlying variability in the data itself. Test folds only

179 contain real heart sounds.

180

181

Figure 2 shows the cross validated ROC curves for the different models. 
Fig. 2a. Model 0

Baseline

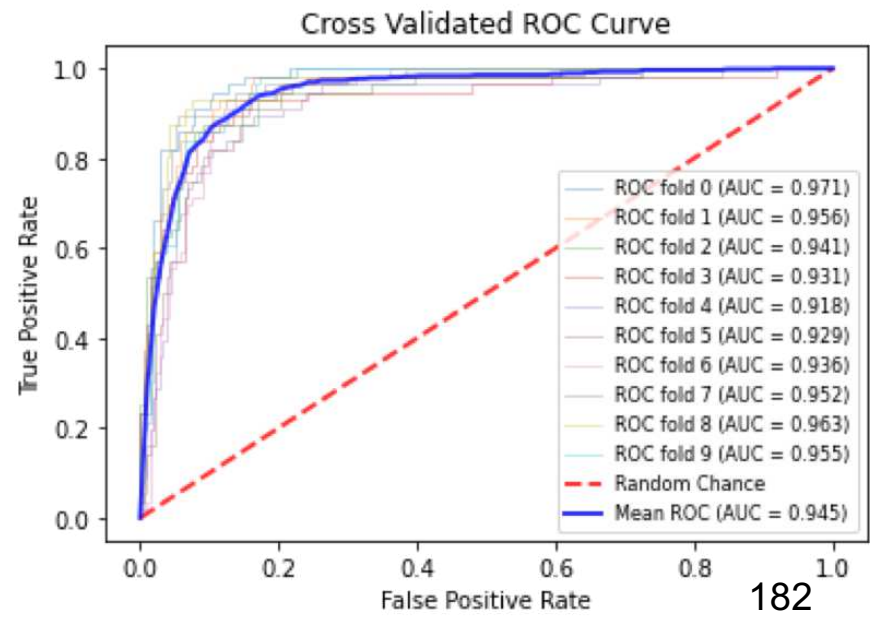

Fig. 2c. Model 2

Noise Injection

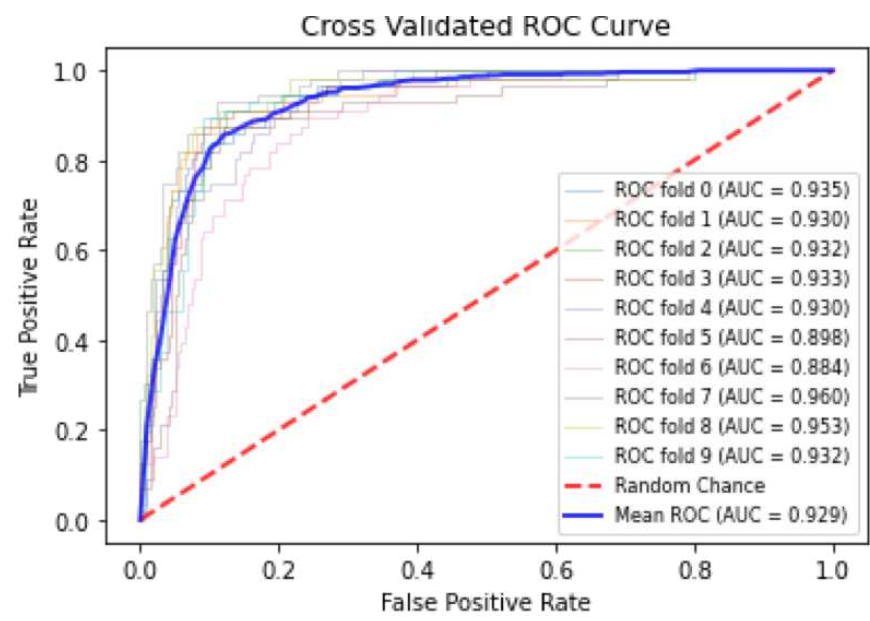

Fig. 2e. Model 3.2

Vertical Flip

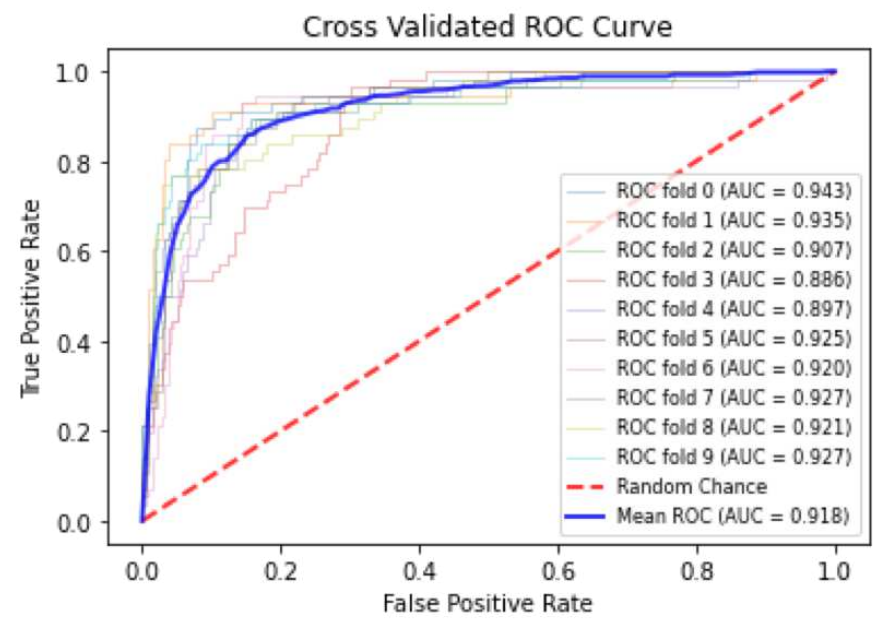

Fig. 2b. Model 1

Pitch Shifting / Time Compression

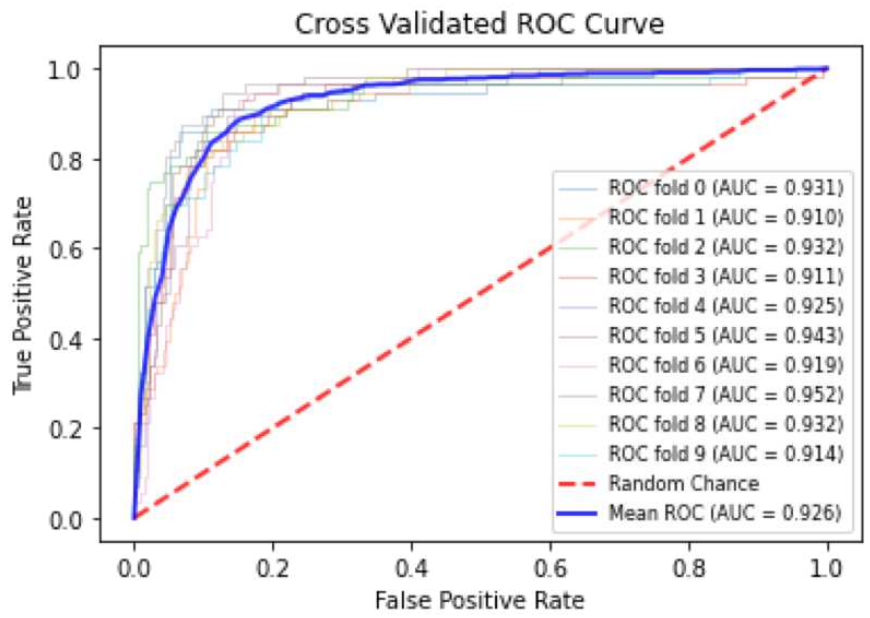

Fig. 2d. Model 3.1

Horizontal Flip

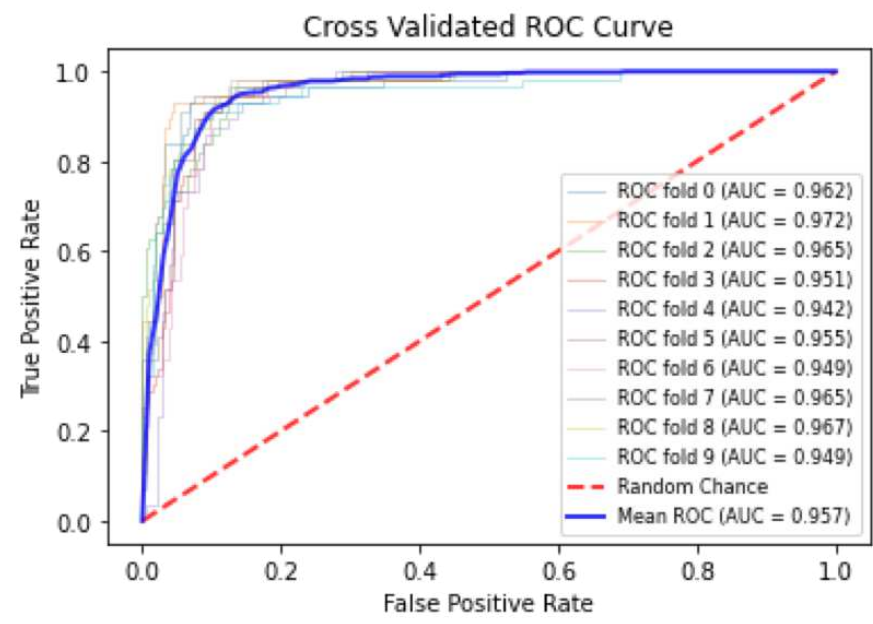

Fig. 2f. Model 4.1

SV Perturbations

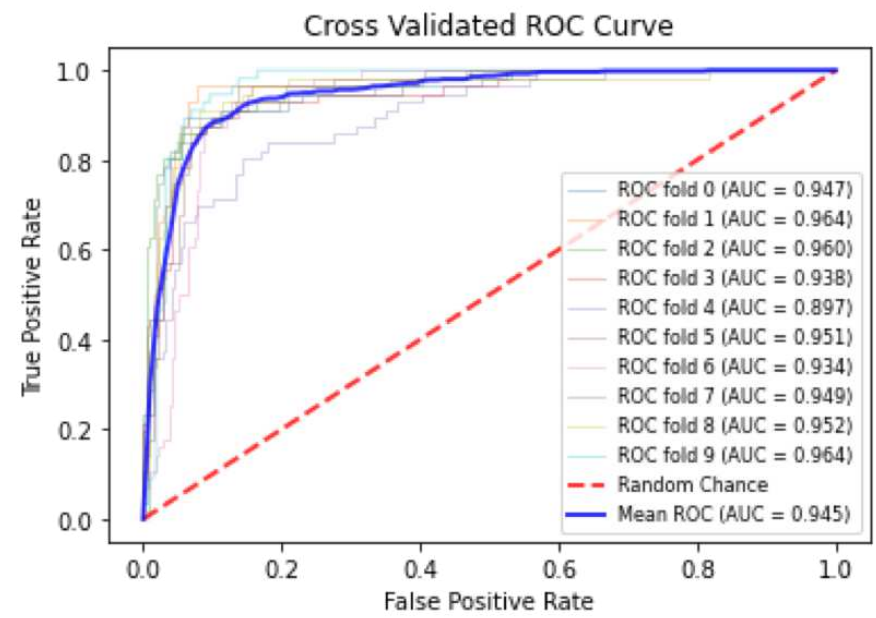


Fig. 2g. Model 4.2 PCA Color Augmentation

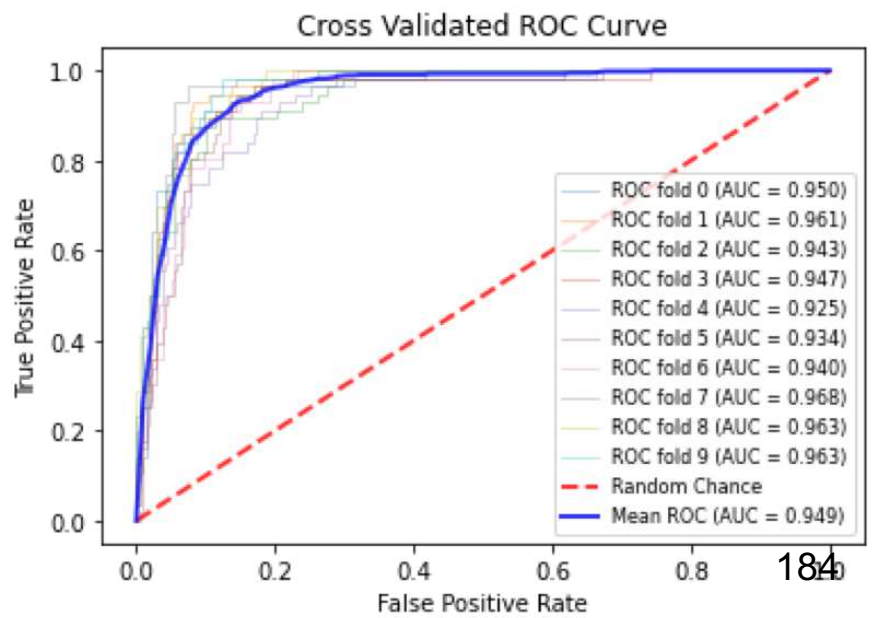

Fig. 2h. Model 4.3 Random Color Filters

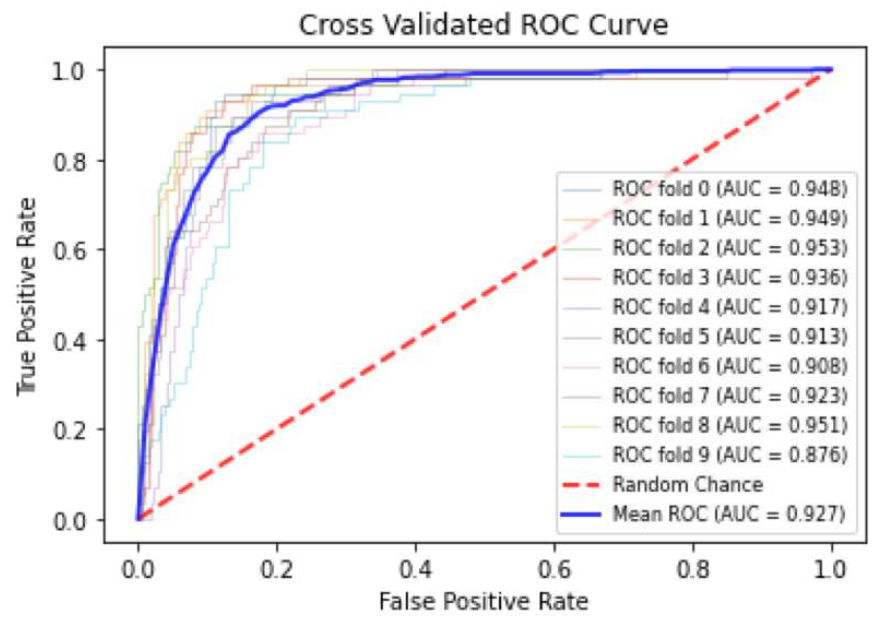

185

186

187

188

189

190

191

192

193

194

195

196

197

198

199

200

201

202

203

204

205

206
Fig. 2i. Model 5

Time and Frequency Masking

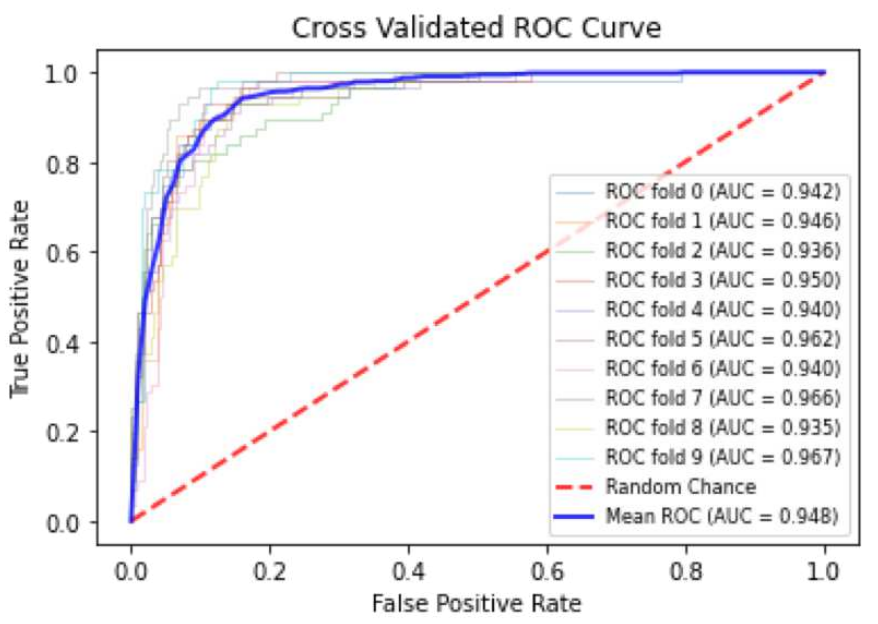

Figure 2: ROC curves for Model 0 (a), Model 1 (b), Model 2 (c), Model 3 (d,e), Model 4 $(\mathbf{f}, \mathbf{g}, \mathrm{h})$, Model 5 (i)

Comparison of the ROC curve for Model 0 , trained on real data only (2a); ROC curve for Model 1 , trained on Mel-Spectrograms of real plus pitch shifted and time stretched/compressed heart sounds (2b); ROC curve for Model 2, trained on Mel-Spectrograms of real plus noise injected heart sounds (2c); ROC curves for Models 3.1 and 3.2, trained on real and horizontally flipped Mel-Spectrograms (2d), and real and vertically flipped Mel-Spectrograms (2e); the ROC curves for Model 4.1, 4.2, and 4.3, trained on real and saturation/value transformed images (2f), real and multi-color transformed Mel-Spectrograms $(2 \mathrm{~g})$, real and PCA color augmented MelSpectrograms (2h); and the ROC curve for Model 5, trained on real and frequency/time masked Mel-spectrograms (2i).The dotted red line represents the no-discrimination line.

Table 1 is a numerical summary of the performance of each model. 
Table 1. Average performance of each model according to accuracy, sensitivity,

\begin{tabular}{|c|c|c|c|}
\hline & Accuracy $( \pm S t d e v)$ & $\begin{array}{c}\text { Specificity } \\
( \pm \text { Stdev) } \\
\text { (at } 90 \% \text { Sensitivity) }\end{array}$ & ROC AUC $( \pm S t d e v)$ \\
\hline $\begin{array}{l}\text { Model } 0 \\
\text { Baseline }\end{array}$ & $89.7 \%(1.7)$ & $86.6 \%(3.8)$ & $0.945(0.016)$ \\
\hline $\begin{array}{l}\text { Model } 1 \\
\text { Pitch/time alterations }\end{array}$ & $88.2 \%(2.4) \downarrow$ & $82.3 \%(4.7) \downarrow$ & $0.926(0.013) \downarrow$ \\
\hline $\begin{array}{l}\text { Model } 2 \\
\text { Noise Injection }\end{array}$ & $88.6 \%(2.1) \downarrow$ & $82.2 \%(6.2) \downarrow$ & $0.929(0.021) \downarrow$ \\
\hline $\begin{array}{l}\text { Model } 3.1 \\
\text { Horizontal Flip }\end{array}$ & $90.2 \%(1.8) \uparrow$ & $90.8 \%(2.7) \uparrow$ & $0.957(0.009) \uparrow$ \\
\hline $\begin{array}{l}\text { Model } 3.2 \\
\text { Vertical Flip }\end{array}$ & $89.2 \%(2.7) \downarrow$ & $79.5 \%(6.9) \downarrow$ & $0.919(0.017) \downarrow$ \\
\hline $\begin{array}{l}\text { Model } 4.1 \\
\text { SV Perturbations }\end{array}$ & $90.6 \%(1.7) \uparrow$ & $80.3 \%(26.9) \downarrow$ & $0.946(0.019) \uparrow$ \\
\hline $\begin{array}{l}\text { Model } 4.2 \\
\text { PCA Color Augmentation }\end{array}$ & $89.2 \%(2.2) \downarrow$ & $87.8 \%(4.3) \uparrow$ & $0.949(0.014) \uparrow$ \\
\hline $\begin{array}{l}\text { Model } 4.3 \\
\text { Random Color Filters }\end{array}$ & $87.4 \%(3.0) \downarrow$ & $81.4 \%(7.0) \downarrow$ & $0.927(0.024) \downarrow$ \\
\hline $\begin{array}{l}\text { Model } 5 \\
\text { Time/Frequency Masking }\end{array}$ & $89.5 \%(1.7) \downarrow$ & $86.2 \%(5.1) \downarrow$ & $0.948(0.012) \uparrow$ \\
\hline
\end{tabular}

\section{Discussion}

213 In summary, our objective was to identify the optimal forms of data augmentation for the binary

214 classification of PCG signals using their spectral image representation. Our baseline CNN

215 model achieved specificity of $86.6 \%$ at $90 \%$ sensitivity, and a ROC AUC of 0.95 , which makes it

216 comparable to state-of-the-art ${ }^{30,31}$. As previously discussed, one of the unique challenges of

217 heart sound augmentation is that the generated samples must fulfill certain "physiological

218 constraints" to remain meaningful. More explicitly, the rate, rhythm, and pitch of cardiac sounds

219 are bounded within a narrow range. Values that fall outside of these limits would be unrealistic, 
220 and hence detract from the classification. Additionally, the original spectral components of the

221 heart sounds must be maintained to ensure that a normal sound does not become pathological.

222 The presence or absence of frequency components like murmurs, rubs, S3, or S4 gallops

223 should be preserved through these transformations. Secondly, the "spectrogram constraint"

224 stems from the fact that spectrograms and photographs fundamentally convey different

225 information along their respective dimensions. Image data augmentation methods can work for

226 spectral images only if they correlate with realistic physical variations in the sound.

228 The data augmentation method that satisfied both the "physiological constraint" and the 229 "spectrogram constraint" improved model performance, while all the data augmentation 230 methods that failed to satisfy at least one of the constraints worsened model performance in 231 some respect, experimentally supporting our theoretical framework. We provide a rationale for

232 why each data augmentation method either improved, did not effect, or worsened model 233 performance using our framework below.

235 The first augmentation method was pitch shifting and time stretching/compressing. Since this 236 augmentation is done at the audio level, the "spectrogram constraint" does not apply. Natural 237 pitch variations reflect different anatomical variations of the heart including differing myocardium 238 wall thickness, body fat/water composition, patient bone/rib structure, and the actual heart size, 239 all of which may lead to variabilities in heart sound attenuation. The data augmentation 240 technique of pitch shifting aims to capture these natural variations. There is also variability in

241 how fast the heart beats. Time stretching and compressing represents heart sounds at different

242 heart rates, such as in tachycardia or bradycardia. Although pitch shifting and time

243 stretching/compressing as data augmentation techniques reflects possible physiological

244 variations, experimentally we see worsening model performance when these data augmentation

245 techniques are applied. At first this seems to contradict our theoretical framework because the 
246 "physiological constraint" is supposedly satisfied. However, if we considered that the natural

247 heart sound exists within a very narrow physiological range, it is likely that the upper and lower

248 limits of our pitch shifting, and time stretching/ compressing may have pushed the audio outside

249 the normal physiological range. Thus, the "physiological constraint" was not actually satisfied

250 because our augmentation techniques created sounds that would never exist clinically, which is

251 consistent with the worsening model performance.

253 The second augmentation method was noise injection. Noise injection has a regularization

254 effect that can improve model performance by reducing overfitting and is a widely used audio

255 data augmentation method for improving model performance. This augmentation is also done at

256 the audio level, so again the "spectrogram constraint" does not apply. Despite the known ability

257 of noise injection for improving model performance, we observe that noise injection actually

258 worsens model performance for heart sound spectral image classification. This can be

259 understood from the fact that the fundamental difference between normal and abnormal heart

260 sounds is that the latter has additional frequency components (murmurs, rubs, S3 gallops, S4

261 gallops). By definition, noise injection is the act of introducing new frequency components to an

262 audio file. Thus, noise injection is essentially converting normal heart sounds into abnormal

263 heart sounds. Noise injection fails to satisfy the "physiological constraint" because it ruins the

264 distinction that separates normal and abnormal heart sounds.

265

266 The third augmentation method is flipping the spectrogram image. Horizontal flipping improved

267 model performance on all three counts, while vertical flipping worsened model performance on

268 all three counts. This is explained by the fact that information conveyed by sound is encoded in

269 the frequency domain, which is represented on the y-axis of spectrogram images. This is an

270 important distinction from traditional images, where the y-axis represents a physical distance.

271 Although vertical flipping has been shown to be an effective augmentation technique for 
272 improving model performance on many image datasets such as ImageNet and CIFAR-10 32

273 (which consist of images of commonplace objects like dogs, cats, cars, etc.), a vertical flip is not

274 appropriate for a spectrogram image. Transformations of the $y$-axis of spectrograms would

275 scramble the frequency content of the sound, rendering any meaningful information that was

276 encoded in the sound to be lost. A vertical flip has no physical correlation, and so does not

277 satisfy the "spectrogram constraint." In fact, the vertical flip worsened model performance the

278 most out of all the data augmentation techniques explored, underscoring the importance of not

279 distorting the $y$-axis of spectrogram images. Horizontal flipping leaves the frequency axis intact,

280 so it satisfies the "spectrogram constraint". A horizontal flip alters the temporal relationships of

281 the frequency components, but as discussed above, a normal and pathological heart sound

282 mostly contain the same frequency components (S1, S2, systole, diastole). The major difference

283 is the presence or absence of other frequency components such as murmurs. It is not so much

284 the temporal relationship of these frequency components with each other that help discern a

285 normal heart sound from a pathological one. Thus, horizontal flips satisfy the "physiological

286 constraint" as well, and experimentally we observe that horizontal flips improve model

287 performance the most out of all data augmentation methods explored. Horizontal flipping as a

288 data augmentation technique is most likely unique to heart sound spectral images compared to

289 many other audio classification problems that represent sound as spectral images, owing to the

290 rhythmic nature of heart sounds. In other audio classification tasks such as speech recognition,

291 the temporary relationship of the different frequency components is important, and thus a

292 horizontal flip would most likely hinder model performance.

294 The next set of data augmentation methods (methods 4.1,4.2, and 4.3) are various color space

295 transformations. Although these transformations do not distort the frequency axis of the

296 spectrogram, it is important to keep in mind the role of color as an additional dimension in

297 spectrogram images. In a regular photo, color represents the wavelength of light reflecting off an 
298 object. In a spectrogram, color represents the loudness/intensity of the signal measured in 299 decibels. Factors that contribute to the natural variation in heart sound amplitudes (i.e. how loud 300 the heart sound is) include the size and position of the heart in the mediastinum, the presence 301 of fluid within or fibrous thickening of the pericardium, and the position and extent of aeration of

302 the lungs. For example, heart sounds are usually loudest at the apex where the heart is in direct 303 contact with the anterior wall of the thorax. Younger patients tend to have louder heart sounds 304 due to elastic and thin chest walls, whereas older patients tend to have quieter heart sounds 305 due to stiffer and thicker chest walls. Heart sounds are louder when the patient is in full 306 expiration, and quieter when the patient is in full inspiration. The data augmentation technique of 307 color space transformations aims to capture these variations. Experimentally, we observe that 308 SV (method 4.1) and PCA (method 4.2) did not lead to statistically significant improvements in 309 model performance, while adding random color filters (method 4.3) unequivocally worsened 310 model performance. Neither SV (method 4.1) and PCA (method 4.2) introduces temporal or 311 spectral distortions to the underlying image, thus satisfying the "spectrogram constraint."

312 However, specificity post-SV augmentation worsened significantly, likely due to the 313 unconstrained shading changes to the spectrogram, which translates to drastic alterations of 314 loudness/intensity at the audio level. The model is less able to identify "normal" heart sounds 315 due to the sheer amount of unnatural variations in the training set that were labeled as normal 316 based on the lack of murmurs. In contrast, incorporation of PCA data in the training set 317 improved sensitivity and ROC AUC at the expense of a minor decrease in accuracy, and overall 318 appears to be the second-best data augmentation method for cardiac analysis next to horizontal 319 flip. At root, PCA establishes new features, known as "principal components," from the original 320 dataset. The goal is to compress the initial input dimensionality without compromising the most 321 valuable information that were conveyed. Alterations along these "principal components" 322 accomplish two objectives. First, they enrich the image along the axes of natural variation, 323 which are by definition where the maximum between-sample variabilities exist. Second, since 
324 changes are made at the color level, the underlying object invariance is maintained, which

325 preserves the temporal and spectral properties of the original spectrograms. While intensity

326 changes are unpredictable in SV because they are randomly generated, PCA's perturbations

327 were derived mathematically, though still unconstrained by human physiological limits.

328 Therefore, PCA suffers a similar pitfall as SV, though the detrimental effects are arguably much

329 more blunted because the "physiologic constraint" is satisfied to a greater extent.

331 In contrast to the previous two techniques, random color filters entirely shift the hues outside the

332 scope of our predetermined color-axis (i.e. orange). This may work for images of commonplace

333 objects like cars, which can be observed in a wide variety of colors, but these augmentations

334 are nonsensible for our heart sound spectrograms as they have no associated physical

335 meaning. The spectrogram constraint is severely violated, and experimentally we observe that

336 multicolor filters worsen model performance to the largest degree on all three counts. It is also

337 important to note that in addition to the natural variations in heart sounds amplitudes, changes

338 in amplitude may also reflect clinically relevant information. Pathological conditions such as

339 cardiac tamponade classically lead to diminished heart sounds. Pleural effusions, subcutaneous

340 edema, pneumothorax, and chronic obstructive pulmonary diseases (COPD) such as

341 emphysema would also muffle heart sounds, although in these conditions the heart itself would

342 be considered healthy. Similar to noise injection, alterations in heart sound amplitude could

343 potentially blur the distinction between normal and abnormal heart sounds, which would worsen

344 model performance. Epidemiologically, distant heart sounds from tamponade, pneumothorax, or

345 COPD that is severe enough to muffle heart sounds are much rarer than murmurs. The majority

346 of abnormal heart sounds in our data set are characterized by murmurs rather than distant heart

347 sounds, explaining why amplitude perturbations did not have as much as a deleterious effect

348 compared to noise injections. 
350 The fifth augmentation method is time and frequency masking. Masking induces partial

351 information loss at random points in the time and frequency domain. We surmise that masking

352 has a similar effect to the regularization technique of dropout, where randomly selected neurons

353 are ignored during training. However, in clinical practice, sudden quiescent periods occur in

354 diseases such as AV heart block, cardiac arrest, or sick sinus syndrome. The original labels are

355 preserved, so images that sprung from masking of normal spectrograms are still labeled as

356 normal, despite the introduction of sudden pauses. Hence, masking does not satisfy the

357 "physiologic constraint" and we observe model performance is not improved. Unlike noise

358 injection and similar to amplitude changes, this type of pathological heart sound is relatively

359 rare, thus there is no drastic reduction in performance. This stands in contrast to the state-of-the

360 art results that masking has achieved in automated speech recognition ${ }^{33}$, further illustrating the

361 distinction between clinical sound analysis and traditional audio processing.

362

363 III. Conclusions

364 Our experimental results corroborate our theoretical framework for thinking about heart sound 365 spectrogram classification. Methods that violated the "spectrogram constraint", such as vertical 366 flipping and applying random color filters, worsened model performance by the greatest extent. 367 Among the methods that did not violate the "spectrogram constraint", the degree to which the 368 "physiological constraint" was adhered to correlated with how much model performance 369 improved or worsened. Noise injection is not a safe operation because the fundamental 370 distinction between normal and abnormal heart sounds is blurred since the majority of abnormal 371 heart sounds (murmurs, gallops, rubs) are just normal heart sounds with additional frequency

372 components. Amplitude variation (via sensible color space transformations) and masking are 373 also limited by fact that the distinction between normal and abnormal heart sounds are blurred:

374 heart sounds with decreased amplitudes can be found in diseases such as cardiac tamponade, 375 and heart sounds with quiescent periods can be found in disease such as AV block. However, 
376 these augmentation methods are less fatal compared to noise injection because

377 epidemiologically these heart sounds are much rarer, explaining why we did not observe a

378 drastic reduction in model performance compared to noise injection. Pitch shifting and time

379 stretching/compressing worsened model performance most likely because the alterations were

380 outside physiological ranges. There is potential for this augmentation method to work but given

381 that heart sounds naturally exist within a narrow physiologic range, future work includes

382 precisely defining these boundaries. Interestingly, horizontal flipping is not actually rooted in any

383 true physiological variation but has proven to be the superior data augmentation method.

384 Horizontal flipping is able to create variation in the data without unnatural variations (such as at

385 the extreme ends of pitch and time alterations) or run the risk of transforming normal sounds

386 into abnormal sounds (such as with amplitude variations or masking). The "physiological

387 constraint" and "spectrogram constraint" can be used as a guide for theory crafting future data

388 augmentation methods for heart sound classification based on their spectral image. Moreover,

389 the ideas behind the "physiological constraint" can be extended to related works seeking to

390 classify heart sounds, while the ideas behind the "spectrogram constraint" can be extended to

391 related work using spectrograms to classify audio.

393 In conclusion, there is value in data augmentation if done correctly, particularly for binary 394 classification of PCG signals, and most likely for other medical classification problems as well.

395 By synthetically generating samples using simple transformations, we can expand on the 396 existing reservoir of patient data, and further enrich the documentation of select pathological 397 conditions, which may be rare in nature and difficult to obtain. Machine learning models are 398 increasingly used to streamline the repetitive processes in healthcare, such as initial screening, 399 preliminary classifications, triage, patient sorting, and specialist recommendations. Data 400 augmentation is a method that has shown utility in improving model performance in cardiac 401 sound analysis and should be further explored in these alternative areas as well. In addition, this 
402 study corroborates the idea that models are only as good as the data from which it learns.

403 Disease-appropriate forms of data augmentation are integral to improvements in model

404 performance, and synthetic data is most meaningful when it lies within the scope of human

405 physiology and can accurately mimic clinical findings. Hence, physician input should be

406 considered when creating models, so these tools can be useful and pragmatic both empirically

407 and at the bedside.

408

409 IV. Methods

$410 \quad 4.1$ Data

411 The data in this study was sourced from a publicly available database assembled from the

412 PhysioNet/Computing in Cardiology (CinC) Challenge in 2016 22,23 . The directory contains 3,239

413 recorded heart sounds that range between 5-120 seconds. The sounds were compiled by

414 physicians and research teams across seven countries over the course of a decade ${ }^{22,23}$. Experts

415 in cardiology labelled the heart sounds as either normal or abnormal. Normal sounds are

416 sounds collected from patients with no underlying cardiometabolic conditions. Abnormal sounds

417 are sounds collected from patients with an underlying cardiac pathology, including valvular

418 defects (i.e. mitral prolapse, mitral regurgitation, aortic regurgitation, aortic stenosis and valvular

419 surgery), as well as coronary artery disease ${ }^{22,23}$. Of the recorded heart sounds, 2575 were

420 labeled as normal and the remaining 664 sounds were labeled as abnormal.

421

\section{$422 \quad 4.2$ Pre-Processing}

423 In concordance with a previous study on heart murmur identification ${ }^{24}$, the raw heart sounds

424 were first processed by a third-order Butterworth filter with a passband of $20-500 \mathrm{~Hz}$, which

425 encapsulates the range of normal heart sound and murmur frequencies ${ }^{25}$. All sounds under 8

426 seconds were discarded. Then, the samples were either truncated to 30 -seconds if their length 
427 exceeded that limit, or preserved in their entirety if the length less than 30 -seconds.

428 Subsequently, the amplitudes of the signals were normalized according to equation 1:

$$
X_{\text {norm }}=\frac{X}{\max (|X|)}
$$

430 where $X$ refers to the amplitude of the signal to ensure it is standardized across all recordings.

\subsection{Mel-Spectrogram}

433 The samples are windowed using a Hann window of size 512 and hop length of 256. A 512-

434 point Fast Fourier Transform is applied to each window to generate a spectrogram, which

435 depicts frequency over time. The amplitude of each frequency component is encoded in color.

436 The amplitude axis is converted to the $\mathrm{dB}$ scale, with the maximum amplitude serving as the

437 reference point and given a value of $0 \mathrm{~dB}$. The frequency axis is transformed onto the Mel scale,

438 which is characterized by equation 2 ,

$$
M e l=2595 * \log \left(1+\frac{f}{500}\right)
$$

440 where $f$ is frequency in $\mathrm{Hz}$.

441

442 The resulting Mel-spectrogram images are standardized by rescaling each image to be of size

$443100 \times 180$ using bicubic interpolation. Figure 3 shows representative examples of the final Mel-

444 spectrogram images. 

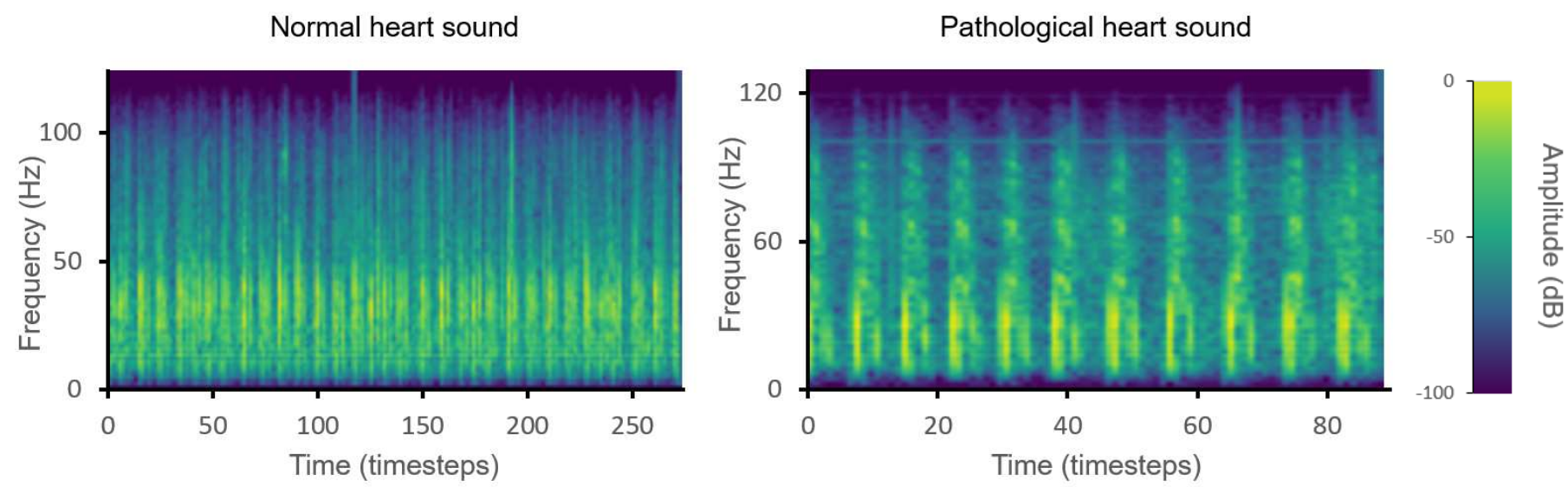

Figure 3: Representative Mel-spectrograms of normal heart sound (left) and pathological heart sound (right)

\subsection{Data Augmentation}

\section{4.4.1 Pitch Shifting and Time Stretching / Compression}

451 To create a synthetic heart sound under method 1, each real heart sound is first randomly pitch

452 shifted up or down by $p$ semitones, where $p$ is a randomly chosen integer between 1 and 10 . A 453 semitone is defined as the interval between two adjacent notes in a 12-tone scale. For example, 454 on a musical scale, the interval between $C$ and $C \#$ is one semitone. Then the pitch shifted 455 sound is randomly time stretched/compressed by a factor of $t$, where $t$ is randomly chosen from 456 the uniform distribution [0.5, 2.0]. For example, if $t=2.0$, then a 30 second audio file is stretched 457 to 60 seconds, or if $t=0.5$, then a 30 second audio file is compressed to 15 seconds. The pitched 458 shifted and time stretched/compressed sounds are then converted to Mel-spectrogram images, 459 which are used to supplement the Mel-spectrogram images derived from real heart sounds to 460 train the convolutional neural network. 
463 To create a synthetic heart sound under method 2, additive white Gaussian noises (AWGN) are

464 injected element-wise into the original signal. The amplitude of AWGN is modeled as a

465 Gaussian distribution, with $\mu=0 .{ }^{26}$ The standard deviation of the noise signal is described with

466 the following formula:

467

$$
R M S=\sqrt{\frac{\sum_{i} x_{i}^{2}}{n}}
$$

468 Assuming a signal-to-noise ratio (SNR) of 0 , the required $R M S_{\text {noise }}$ can be approximated by $469 R M S_{\text {signal }}$. Each element of the noise signal is independently sampled from the distribution $470 X \sim N\left(\mu, \sigma^{2}\right)$ where $\mu=0, \sigma=R M S_{\text {signal }}$. The resulting noise signal is summed with the

471 original sample. The synthetic samples are converted to Mel-spectrogram images and

472 combined with the real heart sound Mel-spectrogram database to train the CNN model.

\section{$474 \quad$ 4.4.3 Image Flip}

475 To create synthetic data under method 3.1, each real heart sound is first converted to a Mel476 spectrogram. The images are flipped horizontally, along an imaginary vertical axis that passes 477 through its center, such that a given pixel with coordinate $(x, y)$ will now be situated at (width$478 x-1, y)$. Figure 3 displays an example of the transformation. For method 3.2, the images are 479 flipped vertically along a centered horizonal axis, such that a given pixel with coordinates $(x, y)$ 480 will now be situated at $(x$, height $-y-1)$. Figure 4 shows illustrative examples of a horizontally 481 and vertically flipped spectrogram image. 
Unaltered Mel-spectrogram

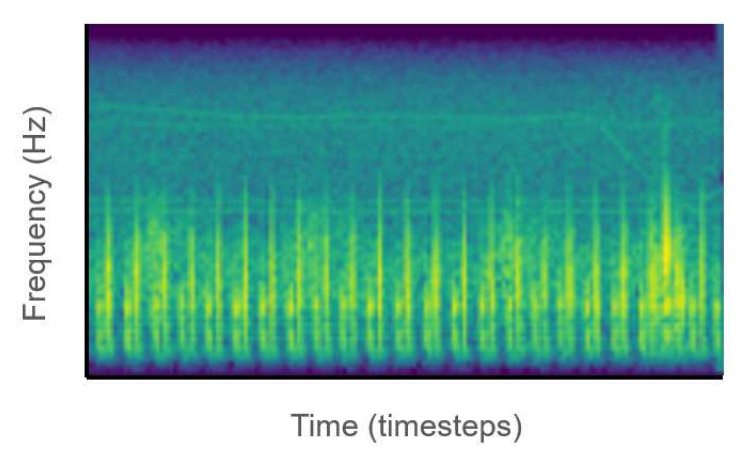

Horizontally Flipped Mel-spectrogram

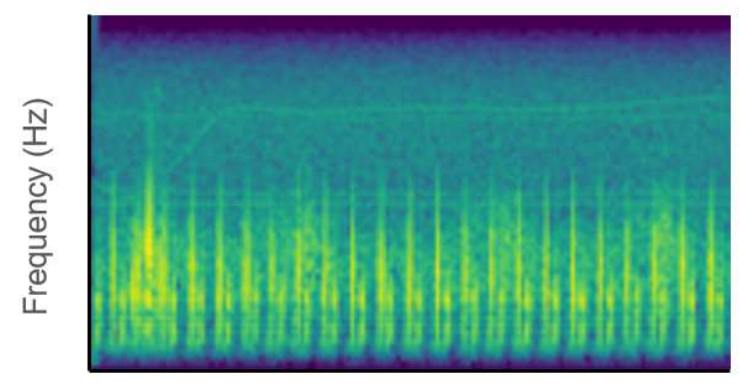

Time (timesteps)
Vertically Flipped Mel-spectrogram

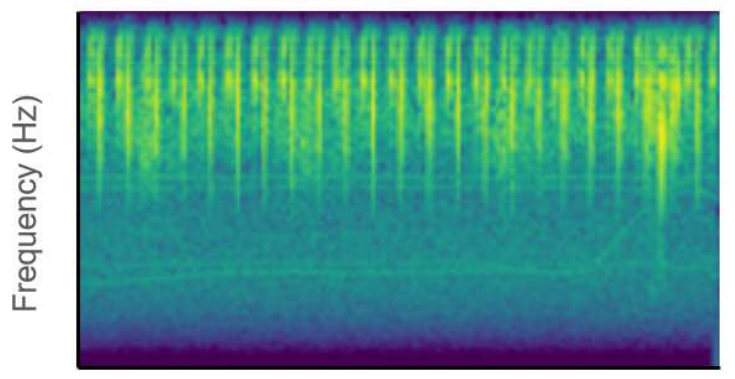

Time (timesteps)

Figure 4: Unaltered Mel-spectrogram (top), horizontally flipped Mel-spectrogram (bottom left), vertically flipped Mel-spectrogram (bottom right)

\subsubsection{Color-Space Transformations}

489 To create synthetic heart sound spectrograms under Method 4, the real heart sounds are first

490 converted into Mel-spectrograms. Then, each image was transformed into their RGB

491 representation, allowing for the extrapolation of other color-space values using pre-established

492 conversion factors and mathematical operations. For example, in an RBG-to-HSV

493 transformation, the red, green, and blue value which range from $([0,255])$ for each pixel, is

494 converted into hue $\left(\left[0^{\circ}, 360^{\circ}\right]\right)$, saturation $([0-100 \%])$, and value/brightness $([0-100 \%])$ using the 495 following formulas ${ }^{27}$ :

$$
R^{\prime}=\frac{R}{255}
$$




$$
G^{\prime}=\frac{G}{255}
$$

$$
B^{\prime}=\frac{B}{255}
$$

$$
C_{\text {max }}=\operatorname{MAX}\left(R^{\prime}, G^{\prime}, B^{\prime}\right)
$$

$$
C_{\min }=\operatorname{MIN}\left(R^{\prime}, G^{\prime}, B^{\prime}\right)
$$

$$
\Delta=C_{\max }-C_{\min }
$$

$$
H=\left\{\begin{array}{c}
60^{\circ} \times\left(\frac{G^{\prime}-B^{\prime}}{\Delta} \bmod 6\right), C_{\max }=R^{\prime} \\
60^{\circ} \times\left(\frac{B^{\prime}-R^{\prime}}{\Delta}+2\right), C_{\max }=G^{\prime} \\
60^{\circ} \times\left(\frac{R^{\prime}-G^{\prime}}{\Delta}+4\right), C_{\max }=B^{\prime} \\
S=\left\{\begin{array}{l}
0, C_{\max }=0 \\
\frac{\Delta}{C_{\max }}, C_{\max } \neq 0
\end{array}\right.
\end{array}\right.
$$

$$
V=C_{\text {max }}
$$

505 Within the scope of color space transformations, we explored three modalities of data

506 augmentation. Method 4.1 created new images from saturation and value perturbations. Method

5074.2 created new images from Principal Component Analysis color augmentation, a method first

508 introduced in Krizhevsky et a ${ }^{28}$. Method 4.3 created new images from applying random color

509 filters.

\section{$511 \quad$ 4.4.4.1 Method 4.1}

512 In Method 4.1, two numbers, $\alpha_{\text {brightness }}$ and $\alpha_{\text {saturation }}$, were randomly drawn from a uniform

513 distribution $X \sim U(a, b)$. Experimentally, it was determined that the $\alpha_{\text {brightness }}$ would be bounded

514 by $\mathrm{a}=0.5$ and $\mathrm{b}=2$, and $\alpha_{\text {saturation }}$ by $\mathrm{a}=0.1$ and $\mathrm{b}=2 . \alpha_{\text {brightness }}$ and $\alpha_{\text {saturation }}$ control the 515 degree of brightness and saturation perturbations, respectively. The merging operation can be 516 described with the following formula: 
518 Brightness alterations were achieved by blending the original image with a pure black image of

519 the same dimensions. Saturation alterations were achieved by blending the original image with

520 a grey-scale image of the same dimensions. The two perturbations were applied sequentially to

521 the original image, and the adjustment factors $\alpha_{\text {brightness }}$ and $\alpha_{\text {saturation }}$ were redrawn for each

522 input spectrogram. Figure 5 shows spectrograms that have undergone saturation and

523 brightness perturbations.

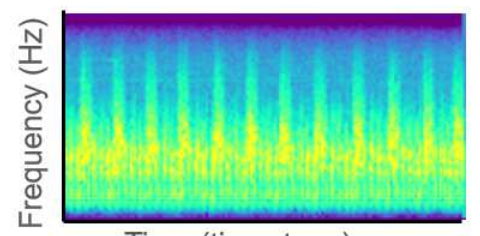

Time (timesteps)

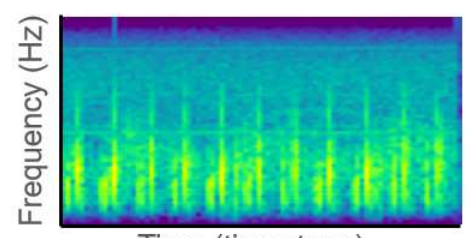

Time (timesteps)

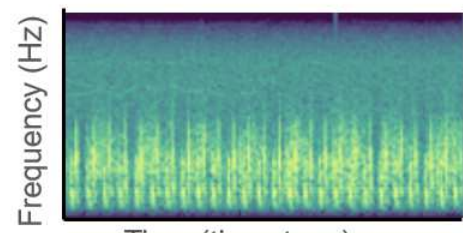

Time (timesteps)

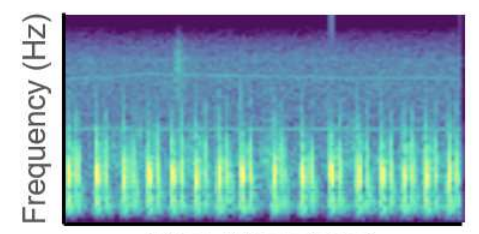

Time (timesteps)

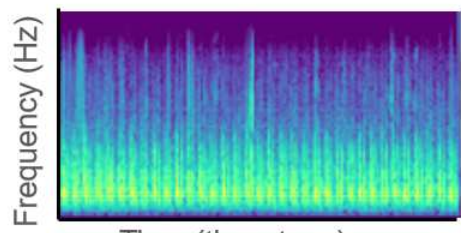

Time (timesteps)

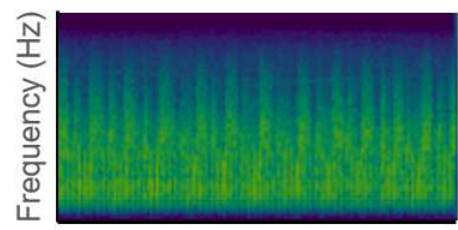

Time (timesteps)

\section{Figure 5: Representative Mel-spectrograms with Saturation Brightness Perturbations}

$526 \quad$ 4.4.4.2 Method 4.2

527 In Method 4.2, as described in Krizhevsky et a/28, we implemented principal component analysis

528 on the unaltered input images, yielding a sorted set of eigenvectors and eigenvalues that are

529 associated with the $3 \times 3$ covariance matrix of the RGB color channels. We then drew a random

530 variable $\alpha$ from the normal distribution $X \sim N\left(\mu, \sigma^{2}\right)$, where $\mu=800, \sigma=10$, and multiplied it to

531 the original eigenvalues. The principal components are scaled by the output from the previous

532 step, and the product is added to the RGB vector of each individual pixel. $\alpha$ is drawn once for

533 each training image. The specific mean and standard deviation values of the perturbation were 
534 chosen experimentally, to intentionally produce more pronounced differences in the output 535 images. Figure 6 shows spectrograms that have undergone PCA color augmentation.

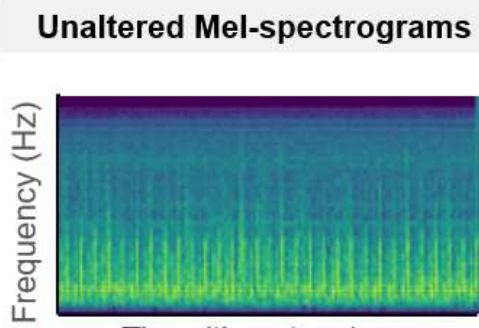

Time (timesteps)

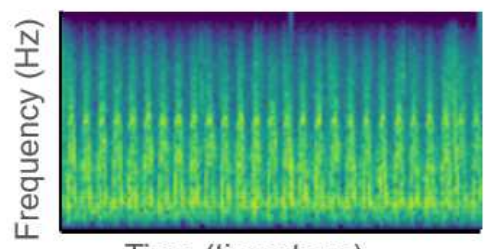

Time (timesteps)
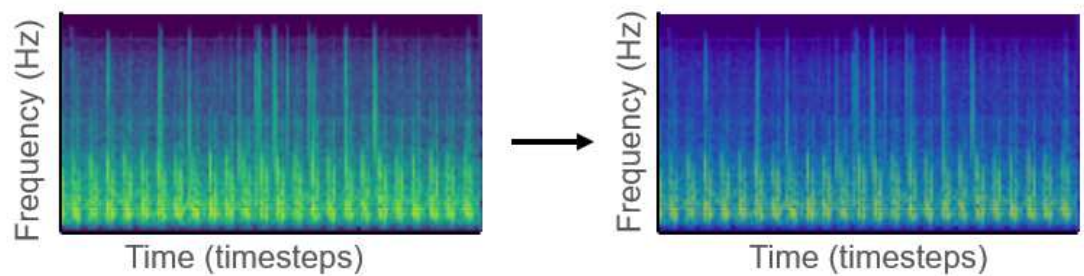

537 Figure 6: Unaltered Mel-spectrograms (Left), same images after Principal Component 538 Analysis (PCA) Color Augmentation (Right) (Data Augmentation Method 4.2)

\subsubsection{Method 4.3}

541 In Method 4.3, we iterated through a library of 150 different color-space conversions using the

542 OpenCV package, effectively generating random color balance perturbations, but preserving the

543 underlying shapes and content of the input images. The transformed Mel-spectrograms are

544 used to supplement the Mel-spectrograms from real heart sounds as additional training data.

$545 \quad$ Figure 7 shows spectrograms with random color filters applied. 


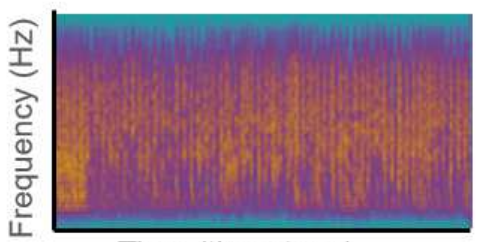

Time (timesteps)

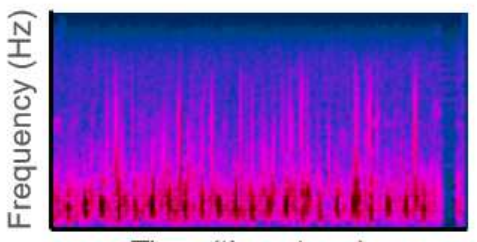

Time (timesteps)

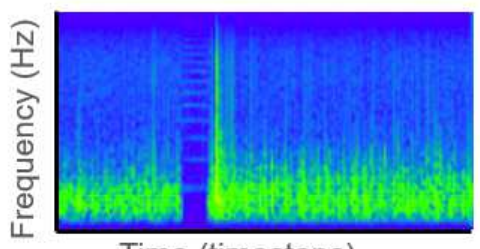

Time (timesteps)

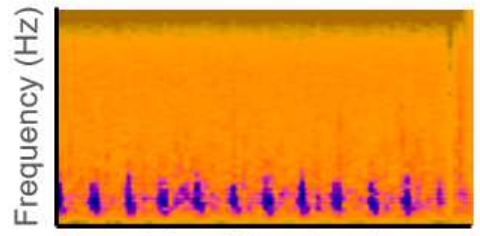

Time (timesteps)

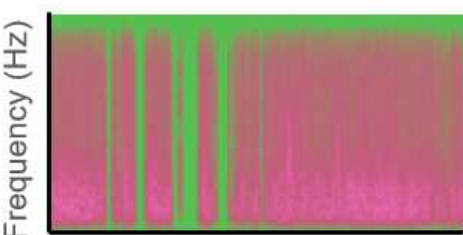

Time (timesteps)

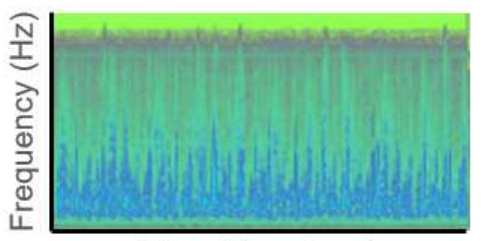

Time (timesteps)

Figure 7: Representative Mel-spectrograms with Random Color Filters

\subsubsection{Time and Frequency Masks}

To create synthetic heart sound data under Method 5 , the real heart sounds are left untouched

552 and converted to Mel-spectrogram images. To the Mel-spectrogram image, three masks are

553 randomly applied in the time domain, and three masks are randomly applied in the frequency

554 domain. In frequency masking, the frequency channels $\left[f_{0}, f_{0}+f\right)$ are masked, where $f$ is

555 randomly chosen from the uniform distribution $[0,20]$, and $f_{0}$ is randomly chosen from $(0, v-f)$,

556 where $v$ is the total number of frequency channels. In time masking, the time steps $\left[t_{0}, t_{0}+t\right)$ are

557 masked, where $t$ is randomly chosen from the uniform distribution [0,20], and $t_{0}$ is randomly

558 chosen from $[0, \boldsymbol{\tau}-t]$, where $\boldsymbol{\tau}$ the total number of time steps. Figure 3 illustrates an example of

559 a transformed Mel-spectrogram. The location of the masks is chosen independently, meaning it

560 is possible for masks to overlap and merge into one larger mask. The transformed Mel-

561 spectrogram images are used to supplement the Mel-spectrogram images derived from real

562 heart sounds to train the convolutional neural network. Figure 8 shows a spectrogram with time 563 and frequency masking applied. 


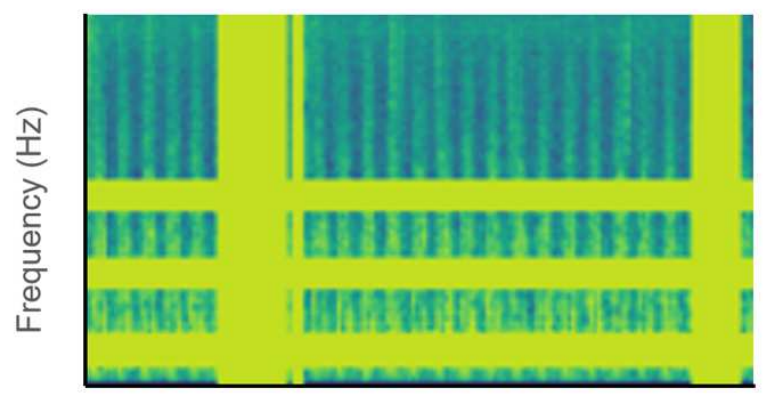

Figure 8: Representative example of time / frequency masked Mel-spectrogram

572 Three masks, as represented by the yellow bars, are randomly applied in the time domain, and 573 three masks are randomly applied in the frequency domain.

\subsection{Convolutional Neural Network}

576 The resulting Mel-spectrograms are treated as images and used to train a convolutional neural 577 network (CNN) for binary classification. A prior study that explored heart sound classification 578 provided an optimized CNN framework that inspired the basis of the CNN architecture used in 579 this study ${ }^{29}$. The convolutional neural network model we built consists of four layers. The first 580 layer is a convolution layer with $323 \times 3$ kernels, each with a stride length of one; the activation 581 function used is a rectified linear (ReLU) activation function.

583 This is followed by a max pooling layer with a filter of size $2 \times 2$ with a stride length of two. The 584 second layer is a convolutional layer with $643 \times 3$ kernels, each with a stride length of one; the 585 activation function used is a ReLU activation function. Similarly, it is followed by a max pooling 586 layer with a filter of size 2x2 with a stride length of two. Padding is not used in any layer. The 587 output from the previous operation is flattened into a one-dimensional feature vector, and then 588 passed to the third layer, a fully connected layer with 64 hidden units. The fourth and final layer 589 is a single neuron with a sigmoid activation function to make the final binary classification. We 
591 performance. Ten epochs are used for training. Figure 9 shows the CNN architecture.

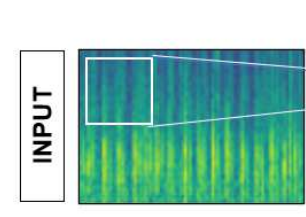

Mel-Spectrogram
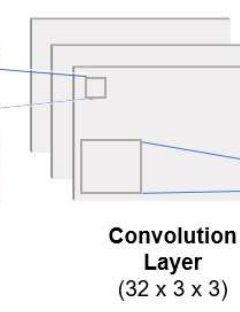

592

593

594

595

596

597

V. List of Abbreviations

Figure 9: Convolutional Neural Network Structure

Illustration of the CNN architecture employed in our study for heart sound classification.

598 AUC: Area under the receiver operating curve

599 ROC: Receiver Operating Curve

$600 \quad$ PCA: Principal component analysis

601 SV: Saturation/Value

602 PCG: phonocardiogram

603 HIPAA: Health Insurance Portability and Accountability Act

604 IRB: Institutional Review Board

605 EHR: Electronic health records

606 COVID-19: Coronavirus disease of 2019

607 AWGN: Additive white Gaussian noises

608 SNR: Signal-to-noise ratio

609 RMS: Root mean squared

610 RGB: Red/Green/Blue 
HSV: Hue/Saturation/Value

612 ReLU: Rectified linear activation function

613 Adam: Adaptive Moment Estimation

614 CIFAR: Canadian Institute For Advanced Research

615 COPD: Chronic obstructive pulmonary diseases

\section{References}

6181 Nielsen $\mathrm{T}$, Molgaard H, Ringsted C, Eika B. The development of a new cardiac auscultation 619 test: how do screening and diagnostic skills differ? Med Teach 2009;online first, DOI:

$62010.1080 / 01421590802572767$.

2 Mangione S. Cardiac auscultatory skills of physicians-in-training: a comparison of three English-speaking countries. Am J Med 2001;110:210-16.

3 Dhuper S, Vashist S, Shah N, Sokal M. Improvement of cardiac auscultation skills in pediatric residents with training. Clin Pediatr (Phila) 2007;46:236-40.

4 Nogueira D. M., Ferreira C. A., Gomes E. F., Jorge A. M. Classifying heart sounds using images of motifs, MFCC and temporal features. Journal of Medical Systems. 2019;43(6):p. 168. doi: $10.1007 / \mathrm{s} 10916-019-1286-5$

5 Hamidi M., Ghassemian H., Imani M. Classification of heart sound signal using curve fitting and fractal dimension. Biomedical Signal Processing and Control. 2018;39:351-359. doi: 10.1016/j.bspc.2017.08.002.

$6 \mathrm{~V}$. Maknickas, A. Maknickas Recognition of normal-abnormal phonocardiographic signals using deep convolutional neural networks and mel-frequency spectral coefficients Physiol. Meas., 38 (8) (2017), pp. 1671-1684

7 Juniati D., Khotimah C., Wardani D. E. K., Budayasa K. Fractal dimension to classify the heart sound recordings with KNN and fuzzy c-mean clustering methods. Journal of Physics Conference Series. 2018;953:p. 012202. doi: 10.1088/1742-6596/953/1/012202.

8 Karar M. E., El-Khafif S. H., El-Brawany M. A. Automated diagnosis of heart sounds using rule-based classification tree. Journal of Medical Systems. 2017;41(4):p. 60. doi: 10.1007/s10916-017-0704-9 autocorrelation feature and diffusion maps. Future Generation Computer Systems. 2016;60:1321. doi: 10.1016/j.future.2016.01.010

10 Zhang W., Han J., Deng S. Heart sound classification based on scaled spectrogram and tensor decomposition. Expert Systems with Applications. 2017;84:220-231. doi:

10.1016/j.eswa.2017.05.014 
655

656

657

658

659

660

661

662

663

664

665

666

667

668

669

670

671

672

673

674

675

676

677

678

679

680

681

682

683

684

685

686

687

688

689

690

691

692

693

694

695

696

697

698

699

700

701

702

703

704
11 Whitaker B. M., Suresha P. B., Liu C., Clifford G. D., Anderson D. V. Combining sparse coding and time-domain features for heart sound classification. Physiological Measurement. 2017;38(8):1701-1713. doi: 10.1088/1361-6579/aa7623.

12 Cheng X., Zhan Q., Wang J., Ma R. A high recognition rate of feature extraction algorithm without segmentation. IEEE 6th International Conference on Industrial Engineering and Applications (ICIEA); 2019; Tokyo, Japan. IEEE; pp. 923-927.

13 Wang P, Lim CS, Chauhan S, Foo JY, Anantharaman V. Phonocardiographic signal analysis method using a modified hidden Markov model. Ann Biomed Eng. 2007 Mar;35(3):367-74. doi: 10.1007/s10439-006-9232-3. Epub 2006 Dec 14. PMID: 17171300.

14 Chauhan S, Wang P, Sing Lim C, Anantharaman V. A computer-aided MFCC-based HMM system for automatic auscultation. Comput Biol Med. 2008 Feb;38(2):221-33. doi:

10.1016/j.compbiomed.2007.10.006. Epub 2007 Nov 28. PMID: 18045582.

15 T. I. Yang and H. Hsieh, "Classification of acoustic physiological signals based on deep learning neural networks with augmented features," 2016 Computing in Cardiology Conference (CinC), Vancouver, BC, 2016, pp. 569-572.

16 Raza, A., Mehmood, A., Ullah, S., Ahmad, M., Choi, G. S., \& On, B. W. (2019). Heartbeat Sound Signal Classification Using Deep Learning. Sensors (Basel, Switzerland), 19(21), 4819. https://doi.org/10.3390/s19214819

17 H. Ryu, J. Park and H. Shin, "Classification of heart sound recordings using convolution neural network," 2016 Computing in Cardiology Conference (CinC), Vancouver, BC, 2016, pp. 1153-1156.

18 B. Bozkurt, I. Germanakis, Y. Stylianou A study of time-frequency features for CNN-based automatic heart sound classification for pathology detection Comput. Biol. Med., 100 (August 2017) (2018), pp. 132-143

19 Halevy A, Norvig P, Pereira F. The unreasonable effectiveness of data. IEEE Intell Syst. 2009;24:8-12.

20 Chen S, Abhinav S, Saurabh S, Abhinav G. Revisiting unreasonable effectiveness of data in deep learning era. In: ICCV; 2017. p. 843-52.

21 Shorten, C., Khoshgoftaar, T.M. A survey on Image Data Augmentation for Deep Learning. J Big Data 6, 60 (2019). https://doi.org/10.1186/s40537-019-0197-0

22 Liu C, Springer D, Li Q, Moody B, Juan RA, Chorro FJ, Castells F, Roig JM, Silva I, Johnson AE, Sye Z, Schmidt SE, Papadaniil CD, Hadjileontiadis L, Naseri H, Moukadem A, Dieterlen A, Brandt C, Tang H, Samieinasab M, Samieinasab MR, Sameni R, Mark RG, Clifford GD. An open access database for the evaluation of heart sound algorithms. Physiological Measurement 2016;37(9)

23 Goldberger A, Amaral L, Glass L, Hausdorff J, Ivanov PC, Mark R, Mietus JE, Moody GB, Peng CK, Stanley HE. PhysioBank, PhysioToolkit, and PhysioNet: Components of a new 
705

706

707

708

709

710

711

712

713

714

715

716

717

718

719

720

721

722

723

724

725

726

727

728

729

730

731

732

733

734

735

736

737

738

739

740

741

742

743

744

745

746

747

748

749

750

751

752

753

754

research resource for complex physiologic signals. Circulation [Online]. 101 (23), pp. e215e220.

24 S. Kang, R. Doroshow, J. McConnaughey and R. Shekhar, "Automated Identification of Innocent Still's Murmur in Children," in IEEE Transactions on Biomedical Engineering, vol. 64, no. 6, pp. 1326-1334, June 2017, doi: 10.1109/TBME.2016.2603787.

25 McGee, S. (2018). Chapter 39 - Auscultation of the Heart: General Principles. EvidenceBased Physical Diagnosis (Fourth Edition). S. McGee. Philadelphia, Elsevier: 327-332.e321.

26 Liu W, Lin W. Additive white Gaussian noise level estimation in SVD domain for images. IEEE Trans Image Process [Internet]. 2013 Mar;22(3):872-83. Available from:

http://www.ncbi.nlm.nih.gov/pubmed/23008255

27 G. Saravanan, G. Yamuna and S. Nandhini, "Real time implementation of RGB to $\mathrm{HSV} / \mathrm{HSI} / \mathrm{HSL}$ and its reverse color space models," 2016 International Conference on Communication and Signal Processing (ICCSP), Melmaruvathur, 2016, pp. 0462-0466, doi: 10.1109/ICCSP.2016.7754179.

28 Krizhevsky A, Sutskever I, Hinton GE. ImageNet classification with deep convolutional neural networks. Commun ACM [Internet]. 2017 May 24;60(6):84-90. Available from:

https://dl.acm.org/doi/10.1145/3065386

29 Zhang W, Han J. Towards heart sound classification without segmentation using convolutional neural network. In: Computing in Cardiology. 2017.

30 M. Tschannen, T. Kramer, G. Marti, M. Heinzmann and T. Wiatowski, "Heart sound classification using deep structured features," 2016 Computing in Cardiology Conference (CinC), Vancouver, BC, 2016, pp. 565-568.

31 Bradley M Whitaker et al 2017 Physiol. Meas. 381701

32 J. Shijie, W. Ping, J. Peiyi and H. Siping, "Research on data augmentation for image classification based on convolution neural networks," 2017 Chinese Automation Congress (CAC), Jinan, 2017, pp. 4165-4170, doi: 10.1109/CAC.2017.8243510.

33 Park, D.S., Chan, W., Zhang, Y., Chiu, C., Zoph, B., Cubuk, E.D., Le, Q.V. (2019) SpecAugment: A Simple Data Augmentation Method for Automatic Speech Recognition. Proc. Interspeech 2019, 2613-2617, DOI: 10.21437/Interspeech.2019-2680.

\section{Declarations}

Ethical approval and consent to participate

Not applicable

Consent for publication

Not Applicable

Availability of data and materials 
755 The datasets used and/or analyzed during the current study are available at

756 https://physionet.org/content/challenge-2016/1.0.0/

Competing interests

759 The authors declare no competing interests.

\section{Funding}

The authors did not receive funding for this study.

\section{Author's Contributions}

G.Z. conceived of the presented idea. G.Z. and Y.C. designed and performed the experiments.

Acknowledgements

769 The authors would like to thank Dr. Patrick Flynn and Dr. George Shih for their support.

770

Author's Information

772 Affiliations

773 Weill Cornell Medicine, New York, NY, 10021, USA

774 George Zhou, Yunchan Chen, Candace Chien

775

776

777

778

779 